\title{
The relationship between anxiety and depression in animal models: a study using the forced swimming test and elevated plus-maze
}

R. Andreatini and L.F.S. Bacellar
Laboratório de Fisiologia e Farmacologia do Sistema Nervoso Central, Departamento de Farmacologia, Universidade Federal do Paraná, Curitiba, PR, Brasil

\section{Correspondence \\ R. Andreatini \\ Laboratório de Fisiologia e \\ Farmacologia do Sistema Nervoso \\ Central \\ Departamento de Farmacologia \\ Centro Politécnico \\ Setor de Ciências Biológicas \\ UFPR \\ Caixa Postal 19031 \\ 81540-970 Curitiba, PR \\ Brasil \\ Fax: + 55-41-226-2042/336-5962 \\ E-mail: andreat@bio.ufpr.br \\ Presented at the XII Annual Meeting of the Federação de Sociedades de Biologia Experimental, Caxambu, MG, Brasil, August 1997.}

Received O ctober 6, 1998 Accepted M ay 25, 1999

\section{Abstract}

The present study evaluated the correlation between the behavior of mice in the forced swimming test (FST) and in the elevated plus-maze (PM). The effect of the order of the experiments, i.e., the influence of the first test (FST or PM) on mouse behavior in the second test (PM or FST, respectively) was compared to handled animals (HAND). The execution of FST one week before the plus-maze (FST-PM, N = 10), in comparison to mice that were only handled (HAND-PM, $\mathrm{N}=10$ ) in week 1 , decreased $\%$ open entries (HAND-PM: $33.6 \pm 2.9$; FST-PM: $20.0 \pm 3.9 ;$ mean \pm SEM; $\mathrm{P}<0.02$ ) and $\%$ open time (HAND-PM: 18.9 \pm 3.3 ; FST-PM: $9.0 \pm 1.9 ; \mathrm{P}<0.03$ ), suggesting an anxiogenic effect. No significant effect was seen in the number of closed arm entries (FST-PM: 9.5 (7.0-11.0); HAND-PM: 10.0 (4.0-14.5), median (interquartile range); $\mathrm{U}=46.5 ; \mathrm{P}>0.10$ ). A prior test in the plus-maze (PMFST) did not change \% immobility time in the FST when compared to the HAND-FST group (HAND-FST: $57.7 \pm 3.9$; PM-FST: $65.7 \pm 3.2$; mean $\pm \mathrm{SEM} ; \mathrm{P}>0.10$ ). Since these data suggest that there is an order effect, the correlation was evaluated separately with each test sequence: FST-PM $(\mathrm{N}=20)$ and PM-FST $(\mathrm{N}=18)$. There was no significant correlation between \% immobility time in the FST and plus-maze indexes (\% time and entries in open arms) in any test sequence ( $\mathrm{r}:-0.07$ to 0.18 ). These data suggest that mouse behavior in the elevated plus-maze is not related to behavior in the forced swimming test and that a forced swimming test before the plus-maze has an anxiogenic effect even after a one-week interval.

The traditional view postulates anxiety and depression as separate and distinct disorders. However, several clinical studies have shown a great overlapping between depression and anxiety states. For example, the comorbidity of depression and panic disor-

\section{Key words}

- Anxiety

- Depression

- Comorbidity

- Animal models der is about $50-75 \%$ (1), while the comorbidity of depression and generalized anxiety disorder is approximately $60 \%$ (2). Surprisingly, this association has received little attention in animal research.

Hilakivi and Lister (3) reported that the 
immobility time of mice in the forced swimming test (FST) was not correlated with their behavior in the elevated plus-maze (PM). On the other hand, Ferré et al. (4) found a significant correlation between struggling time (escape-directed movements) of mice in the forced swimming test and latency to first entry into open arms in the elevated plusmaze test. Furthermore, one factor explaining $47.0 \%$ of the variance appeared in the factorial analysis. In this factor struggling time and latency to enter the open arms showed a positive load, whereas percentage of time and entries into the open arms showed a negative load. Although these results could strengthen the anxiety-depression relationship, no significant correlation was found between the traditional measures in the elevated plus-maze and struggling. More recently, Prasad et al. (5) have found a weak but significant correlation $\left(r^{2}=0.337\right)$ between the behavioral measures of rats submitted to the elevated plus-maze and forced swimming test. This is a complex relationship showing that rats with low levels of anxiety exhibited an inverse correlation between anxiety and depression (the level of anxiety increased as the level of depression decreased, $r=0.73$ ), while rats with high anxiety showed a direct correlation between anxiety and depression (the level of anxiety increased as the level of depression increased, $r=0.42$ ). Moreover, dehydroepiandrosterone decreased behavioral despair only in high-anxiety rats.

Another approach was used by Overstreet et al. (6), who studied the behavior of Maudsley reactive (MR) and Maudsley nonreactive (MNR) rats (high and low defecation rate in the open field, respectively) in the elevated plus-maze and forced swimming test. MR displayed a higher immobility time in the FST and a lower percentage of open time in the plus-maze than MNR and Wistar rats.

Therefore, some studies suggest the existence of an anxiety-depression association
(4-6) while others do not corroborate it (3). However, in the studies described above the models were used with several modifications in comparison to the original reports. For example, Hilakivi and Lister (3) evaluated the immobility of mice in an 8-min test in the forced swimming test, immediately following a 2-min habituation period; Ferré et al. (4) employed the elevated plus-maze apparatus for rats to measure the behavior of mice, and Prasad et al. (5) used the time for the rat to leave a dark closed arm as the anxiety measure and the behavior of the animal in the first swimming test as the depression index; Overstreet et al. (6) employed a single session in the FST. Another common problem of these studies is the lack of control of the order effect.

Thus, the present experiment was designed to study the correlation of behavior of the same mice tested in the elevated plusmaze and in the forced swimming test using the validated procedures, and to determine whether there is an order effect in the test sequence.

Animals were 78 adult albino male mice (20-25 g) of our own breed. They were housed in groups of ten in polypropylene cages with wood shavings as bedding, under controlled conditions of light (12-h light-dark cycle, lights on at 7:00 a.m.) and temperature $\left(22^{\circ} \mathrm{C}\right)$. The animals had free access to water and food throughout the experiment. In the forced swimming test mice were placed in a glass aquarium $(20 \mathrm{~cm}$ in diameter $\times 30 \mathrm{~cm}$ high) containing 6 - $\mathrm{cm}$ deep cold water $\left(24 \pm 1{ }^{\circ} \mathrm{C}\right)$ for $6 \mathrm{~min}$ and immobility time was recorded during the last $4 \mathrm{~min}$. The mouse was judged to be immobile whenever it stopped swimming and remained floating in the water, with its head just above water level. The water was changed after each animal to avoid olfactory cues left by the previous animal. Following the test the animals were dried in a heated enclosure. The depression index is immobility time, so that antidepressive treatment reduces the immobility time $(7,8)$. Thus, 
a higher immobility time indicates a greater depressive state and a lower immobility time a smaller depressive state. In the elevated plus-maze test mice were placed at the center of the plus-maze facing one of the open arms. During a 5-min test period, the following data were recorded: number of entries and the time spent in the open and the enclosed arms, and the total number of arm entries. Anxiolytic compounds selectively increase the percentage of time spent and/or entries into the open arms and, in contrast, anxiogenic compounds selectively decrease the percentage of time spent and/or the percentage of entries into the open arms. These effects are seen without changes in the total number of arm entries (9). The elevated plus-maze apparatus consisted of two open arms $(30 \times 5 \times 0.25 \mathrm{~cm})$ and two enclosed arms $(30 \times 5 \times 15 \mathrm{~cm})$ with an open roof, arranged in such a way that the two arms of each type were opposite to each other. The maze was elevated $60 \mathrm{~cm}$ above floor level. The maze was constructed of black painted wood. Twenty mice were tested first in the forced swimming test and then ( 1 week apart) in the elevated plusmaze (FST-PM) and 18 animals were tested in the inverse order (plus-maze followed by forced swimming test, PM-FST). The two test sequences were used to minimize the influence of an eventual order effect. Thus the results of the same tests performed in the oposite order were compared to verify the possibility of summing of the data. All experiments were carried out between 8:00 and 11:00 a.m. to avoid the influence of circadian rhythms on emotionality. Since the data fit the parametric assumptions (normality of data and homoscedasticity), statistical analysis was performed using parametric methods, i.e., Student $t$-test to compare groups with different sequences of testing and Pearson's correlation to measure correlation between the behaviors of mice in the two models. The exception was the number of closed arm entries which was analyzed by the Mann-Whitney U-test.

There was a decrease in the $\%$ of open arm entries $(\mathrm{t}=-2.438 ; \mathrm{P}<0.02)$ in the forced swimming-elevated plus-maze sequence group (FST-PM: $19.8 \pm 3.0$; mean \pm SEM) when compared to the elevated plus-mazeforced swimming sequence group (PM-FST: $30.1 \pm 3.5)$. There was no significant difference in \% open time (FST-PM: $11.1 \pm 3.2$; PM-FST: $15.5 \pm 2.8 ; \mathrm{t}=-1.004 ; \mathrm{P}>0.10$ ) or in the number of closed arm entries (8.0 (7.0$10.5)$ and 7.0 (5.5-8.0), median (interquartile range), $\mathrm{U}=142.50 ; \mathrm{P}>0.10)$. There was no difference in immobility time between the two sequence groups (FST-PM: $86.7 \pm$ 2.8; PM-FST: $75.5 \pm 6.5$; mean \pm SEM; $\mathrm{t}=$ $1.6529 ; \mathrm{P}>0.10$ ). Thus, since it is not possible to sum the data from the two sequences of testing, correlation analysis was performed separately between the two sequence groups (Table 1). There was no significant correlation and the correlation indexes were very low $(-0.07$ to 0.18$)$.

These results suggest a possible order effect. However, the comparison of the FSTPM results to PM-FST was not adequate because the data were collected at a one week interval, when several variables may have influenced the behavior of the animals. Thus, a second set of experiments was carried out. Forty mice were divided into 4 groups ( $N=10 /$ group): in 2 groups the ani-

Table 1 - Pearson's correlation between mouse behavior in the elevated plus-maze (PM) and forced swimming test (FST).

Trials were conducted one week apart. Sequence of tests: FST-PM: forced swimming test followed by elevated plus-maze; PM-FST: elevated plusmaze followed by the forced swimming test.

FST (\% immobility time)

FST-PM PM-FST

$(\mathrm{N}=20) \quad(\mathrm{N}=18)$

PM

\% open arm time $\quad-0.07 \quad 0.16$

$\begin{array}{lll}\% \text { open arm entries } & 0.02 & 0.18\end{array}$ 
mals were only handled (HAND) (manipulated, marked and weighed) during week 1 and submitted to FST (HAND-FST) or PM (HAND-PM) during week 2; one group was tested in the PM during week 1 and submitted to the FST during week 2 (PM-FST), and one group was submitted to the FST during week 1 and tested in the PM during week 2 (FST-PM).

The results are shown in Figure 1. There was a significant difference between the HAND-PM and FST-PM groups in \% open arm entries (HAND-PM: $33.6 \pm 2.9$; FSTPM: $20.0 \pm 3.9 ;$ mean \pm SEM; $P<0.02$ ) and $\%$ time spent in the open arm (HAND-PM: $18.9 \pm 3.3$; FST-PM: $9.0 \pm 1.9$; $\mathrm{P}<0.03$ ). No significant effect was observed on number of closed arm entries (HAND-PM: 10.0 (4.0-14.5); FST-PM: 9.5 (7.0-11.0), median

Figure 1 - Effect of prior handling (HAND) or test (forced swimming test - FST or elevated plusmaze - PM) on the behavior of mice ( $N=10 /$ group) in the elevated plus-maze ( $A$ and $B$ ) and in the forced swimming test (C). Trials were conducted one week apart. Data represent the mean \pm SEM. ${ }^{*} \mathrm{P}<0.05$ compared to HAND (Student t-test).
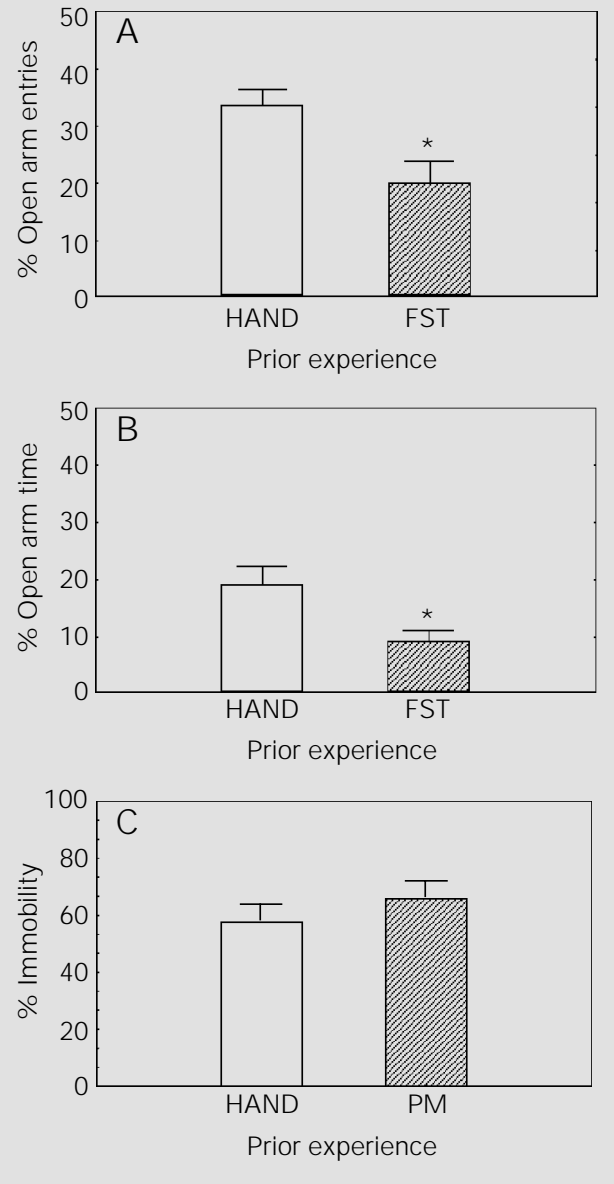

(interquartile range); $\mathrm{U}=46.5 ; \mathrm{P}>0.10$ ). Also, no difference in $\%$ immobility was observed between HAND-FST and PM-FST (HANDFST: $57.7 \pm 3.9$; PM-FST: $65.7 \pm 3.2$; mean \pm SEM; $P>0.10$ ). Taken together with the results of the first set of experiments, this result suggests that there is an order effect of the tests.

Clinical data suggest a relationship between anxiety and depressive states $(1,2)$. However, in the present study we did not detect this correlation in the two most frequently used animal models: the elevated plus-maze and the forced swimming test. Thus, although our results contradict those obtained in some previous studies $(4,5)$, they agree with those reported by Hilakivi and Lister (3). However, the present study was the first to employ the standard (validated) procedures for the animal models used (PM and FST).

These negative results can be explained by the following hypotheses: a) the methodology employed is inadequate to measure the anxiety-depression association; b) the models used are not adequate for this kind of study, and c) the clinical anxiety-depression correlation does not have a neurobiological basis. In clinical studies, the association between these disorders increases with the length of the observation period: it is lower in cross-sectional studies and higher in longitudinal studies (10). Moreover, these disorders are frequently related to chronic stress. Thus, the best methodology may be to perform the tests after a repeated stress period. Indeed it is suggested that the anxiogenic effect observed in the plus-maze after a restraint stress could be a model to study depression-related anxiety (11).

In general, animal models are validated by pharmacological studies, but their validity for another type of study is unknown (12). While the plus-maze has been extensively validated, the FST has been criticized as a model of depression despite its good sensitivity and specificity for antidepressive 
treatments $(8,12)$. Another point that needs consideration is the temporal reliability of the models. Although some studies suggest that these models are stable over time, other studies do not corroborate this view (13). Moreover, all these studies used inappropriate methods to evaluate reliability (e.g., correlation tests), which makes it difficult to draw a conclusion about the real reliability of the models. Apart from the above considerations, the independence between the measures obtained using the plus-maze and the FST, which is suggested by the present study and by a previous study (3), is a valuable indication of the discriminant validity of these models.

The present study also suggests that there is an order effect when the FST is executed before the PM, but not in the inverse order. Previous studies have shown that a broad range of prior stress has an anxiogenic effect on rats and mice tested in the plus-maze, although some negative results were also found. For example, prior immobilization was found to be anxiogenic $(11,14,15)$ or to have no effect $(11,16)$. Furthermore, it was shown in rats that prior stress (habituation to a novel environment and footshock) decreased \% time spent in the open arm, which is correlated with a decrease in the benzodiazepine-like molecules in the amygdala, hippocampus and septum (17). However, in these studies the animals were tested in the plus-maze up to $24 \mathrm{~h}$ after the last stress administration, whereas in the present study this anxiogenic effect was tested after 1 week. It is important to note that this time frame is commonly used in several laboratories to habituate newly arrived animals (e.g., 18). Thus, the present data suggest that a longer period of time may be required to acclimate the animals to laboratory conditions before their use in some models (e.g., plus-maze). Moreover, since the previous stress history may be crucial to determine the animal response to some drugs in the plus-maze behavior (19), some inconsistency in the drug response in the elevated plus-maze can be explained.

Many animal models of depression use prior exposure of animals to stress. For example, it was demonstrated that chronic mild stress induces a state of anhedonia similar to that seen in depressed patients (20). The influence of prior stress on the forced swimming test was evaluated in a few studies. It was shown that chronic (but not acute) immobilization stress reduces the duration of immobility (21), in agreement with the present study which employed only one stress session. On the other hand, another study found that animals exposed to a pre-test session remained immobile for a longer time than those which had not been exposed (20). Moreover, prior exposure to acute stress is necessary to demonstrate the anti-immobility effect of many clinically effective antidepressant drugs (22). However, these studies were conducted on rats and it should be emphasized that the methodologies used for rats and mice were different: there was a 24$\mathrm{h}$ interval between prior swimming stress and testing for rats, while for mice the $2 \mathrm{~min}$ of prior swimming stress was applied immediately before the $4 \mathrm{~min}$ of testing. It would be interesting to study the effect of previous different types of stress on FST behavior. Moreover, although in the present study the PM experience did not modify subsequent FST behavior of drug-naive mice, a previous study found that exposure of animals to PM alters the neurochemical and behavioral (in the second PM trial) effects of chlordiazepoxide (23). Thus, a question to be considered in future experiments is whether a previous PM test alters the drug response of animals submitted to FST.

In conclusion, the data of the present study suggest that mouse behaviors in the elevated plus-maze and in the forced swimming test are not related and that a forced swimming test before the plus-maze has an anxiogenic effect even after a one-week interval. 


\section{References}

1. Keller MB \& Hanks DL (1993). Course and outcome in panic disorder. Progress in Neuro-Psychopharmacology and Biological Psychiatry, 17: 551-570.

2. Wittchen H-U, Zhao S, Kessler RC \& Eaton WW (1994). DSM-III-R generalized anxiety disorder in the national comorbidity survey. Archives of General Psychiatry, 51: 355-364.

3. Hilakivi LA \& Lister RG (1990). Correlations between behavior of mice in Porsolt's swim test and in tests of anxiety, locomotion, and exploration. Behavioral and Neural Biology, 53: 153-159.

4. Ferré $P$, Fernández Teruel $A$, Garcia $E$, Zapata A \& Tobeña A (1994). Struggling and flumazenil effects in the swimming test are related to the level of anxiety in mice. Neuropsychobiology, 29: 23-27.

5. Prasad A, Imamura M \& Prasad C (1997). Dehydroepiandrosterone decreases behavioral despair in high- but not low-anxiety rats. Physiology and Behavior, 62: 1053-1057.

6. Overstreet $\mathrm{DH}$, Rezvani AH \& J anowsky DS (1992). Maudsley reactive and nonreactive rats differ only in some tasks reflecting emotionality. Physiology and Behavior, 52: 149-152.

7. Porsolt RD, Bertin A \& J alfre M (1977). Behavioural despair in mice: a primary screening test for antidepressants. Archives of International Pharmacodynamics, 229: 327-336.

8. Borsini F \& Meli A (1988). Is the forced swimming test a suitable model for revealing antidepressant activity? Psychopharmacology, 94: 147-160.
9. Lister RG (1987). The use of a plus-maze to measure anxiety in the mouse. Psychopharmacology, 92: 180-185.

10. Angst J \& Vollrath M (1989). Relationships between anxiety and depression. In: Kielholz P \& Adams C (Editors), The Kaleidoscope of Anxiety State. Deustcher Ärzte-Verlag GmgH, Colônia, 78-99.

11. McBlane J \& Handley SL (1994). Effects of two stressors on behaviour in the elevated X-maze: preliminary investigation of their interaction with 8-OH-DPAT. Psychopharmacology, 116: 173-182.

12. Willner $P$ (1984). The validity of animal models of depression. Psychopharmacology, 83: 1-16.

13. Lister RG (1990). Ethologically based animal models of anxiety disorders. Pharmacology and Therapeutics, 46: 321-340.

14. Guimarães FS, Del Bel EA, Padovan CM, Mendonça Netto $\mathrm{S} \&$ Titze de Almeida $\mathrm{R}$ (1993). Hippocampal 5-HT receptors and consolidation of stressful memories. Behavioural Brain Research, 59: 133-139.

15. Martijena ID, Calvo N, Volosin M \& Molina VA (1997). Prior exposure to a brief session facilitates the occurrence of fear in response to a conflict situation: behavioral and neurochemical correlates. Brain Research, 752: 136-142.

16. Falter U, Gower AJ \& Gobert J (1992). Resistance of baseline activity in the elevated plus-maze to exogenous influences. Behavioural Pharmacology, 3: 123128.

17. Da Cunha C, Levi De Stein M, Wolfman C, Koya R, Izquierdo I \& MedinaJ H (1992). Effect of various training procedures on performance in an elevated plus-maze: possible relation with brain regional levels of benzodiazepine-like molecules. Pharmacology, Biochemistry and Behavior, 43: 677-681.

18. Trullas R \& Skolnick P (1993). Differences in fear motivated behaviors among inbred mouse strains. Psychopharmacology, 111: 323-331.

19. Rodgers RJ , Cao B- , Dalvi A \& Holmes A (1997). Animal models of anxiety: an ethological perspective. Brazilian J oumal of Medical and Biological Research, 30: 289304.

20. Willner $P$ (1995). Animal models of depression. In: Gessa G, Fratta W, Pani L \& Serra G (Editors), Depression and Mania: From Neurobiology to Treatment. Raven Press, New York.

21. Platt J E \& Stone EA (1982). Chronic restraint stress elicits a positive antidepressant response on the forced swim test. European J ournal of Pharmacology, 82: 179-181.

22. Borsini F, Lecci A, Sessarego A, Frassine R \& Meli A (1989). Discovery of antidepressant activity by forced swimming test may depend on pre-exposure of rats to a stressful situation. Psychopharmacology, 97: 183-188.

23. File SE, Andrews N, Wu PY, Zharkovsky A \& Zangrossi H (1992). Modification of chlordiazepoxide's behavioural and neurochemical effects by handling and plusmaze experience. European J ournal of Pharmacology, 218: 9-14. 